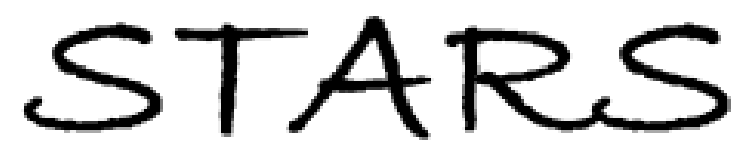

University of Central Florida

STARS

$1-1-1997$

\title{
Exclusion Statistics For Fractional Quantum Hall States On A Sphere
}

\author{
S. B. Isakov \\ G. S. Canright \\ M. D. Johnson \\ University of Central Florida
}

Find similar works at: https://stars.library.ucf.edu/facultybib1990

University of Central Florida Libraries http://library.ucf.edu

This Article is brought to you for free and open access by the Faculty Bibliography at STARS. It has been accepted for inclusion in Faculty Bibliography 1990 s by an authorized administrator of STARS. For more information, please contact STARS@ucf.edu.

\section{Recommended Citation}

Isakov, S. B.; Canright, G. S.; and Johnson, M. D., "Exclusion Statistics For Fractional Quantum Hall States On A Sphere" (1997). Faculty Bibliography 1990s. 1949.

https://stars.library.ucf.edu/facultybib1990/1949

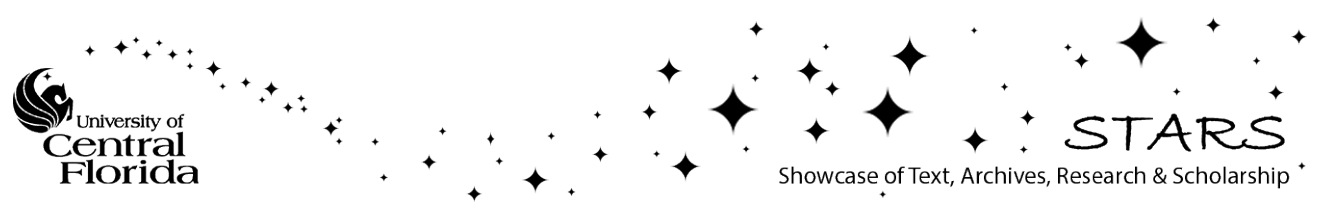




\title{
Exclusion statistics for fractional quantum Hall states on a sphere
}

\author{
S. B. Isakov \\ Center for Hbyere Studier, Drammensveien 78, Oslo, Norway \\ and Department of Physics, University of Oslo, P.O. Box 1048 Blindern, 0316 Oslo, Norway \\ G. S. Canright \\ Center for Hbyere Studier, Drammensveien 78, Oslo, Norway \\ and Department of Physics, University of Tennessee, Knoxville, Tennessee 37996-1200 \\ M. D. Johnson \\ Department of Physics, University of Central Florida, Orlando, Florida 32816-2385
}

(Received 12 November 1996)

\begin{abstract}
We discuss exclusion statistics parameters for quasiholes and quasielectrons excited above the fractional quantum Hall states near $\nu=p /(2 n p+1)$. We derive the diagonal statistics parameters from the ("unprojected") composite fermion (CF) picture. We propose values for the off-diagonal (mutual) statistics parameters as a simple modification of those obtained from the unprojected CF picture, by analyzing finite system numerical spectra in the spherical geometry. [S0163-1829(97)04812-1]
\end{abstract}

Excitations with a fractional charge - quasiholes (QH's) and quasielectrons (QE's) - arising in the fractional quantum Hall $(\mathrm{FQH})$ effect $^{1}$ have been shown to obey fractional exchange statistics, i.e., they are anyons. ${ }^{2}$ The latter concept is two dimensional. However, the system in the FQH effect is restricted to the lowest Landau level (LL), which makes it effectively one dimensional. In this case an algebraic definition of fractional statistics in one dimension ${ }^{3}$ applies to anyons. ${ }^{4}$ One more definition of fractional statistics, exclusion statistics (ES), which explicitly specifies the dimension of the many-particle Hilbert space, was introduced and applied to QH's and QE's. ${ }^{5}$ Connection between the two last definitions is established in statistical mechanics: the statistical distribution for one-dimensional fractional statistics ${ }^{6}$ coincides with that for exclusion statistics. ${ }^{7,8}$ The ES counting directly applies to anyons in the lowest LL. ${ }^{9}$ Relation of the thermodynamics of anyons in the lowest LL (Ref. 10) to that of ES was also shown ${ }^{8}$ including mutual statistical interactions. ${ }^{11}$

The ES counting of states implies for the many-particle Hilbert space dimension ( $\alpha=+$ and - refer to QE's and QH's, respectively) ${ }^{5}$

$$
W=\prod_{\alpha}\left(\begin{array}{c}
D_{\alpha}+N_{\alpha}-1 \\
N_{\alpha}
\end{array}\right),
$$

where $D_{\alpha}=d_{\alpha}-\Sigma_{\beta} g_{\alpha \beta}\left(N_{\beta}-\delta_{\alpha \beta}\right)$, and $d_{\alpha}$ are the dimensions of the one-particle Hilbert spaces. ${ }^{9,12}$

Based on heuristic duality arguments, Haldane $e^{5}$ originally proposed the following values for the statistics parameters: $g_{--}=g_{+-}=-g_{++}=-g_{-+}=1 / \mathrm{m}$ for Laughlin $\nu=1 / \mathrm{m}$ states ( $m$ odd). However, analysis of numerical spectra for a small number of electrons on a sphere for states near $\nu=\frac{1}{3}$ led to the conclusion ${ }^{12}$ that $g_{--}=\frac{1}{3}$ but $g_{++}=\frac{5}{3}$. The origin of the asymmetry between $g_{--}$and $g_{++}$was attributed to the interpretation of QE's as hard-core anyons, resulting in $g_{--}=1 / m, g_{++}=2-1 / m$ for Laughlin states. ${ }^{13-15}$ Very re- cently, values for the off-diagonal statistics parameters, $g_{-+}=-g_{+-}=2-1 / \mathrm{m}$, have also been proposed. ${ }^{17}$

In this paper we discuss the ES parameters for quasiparticles for the more general filling factors $\nu=p /(2 n p+1)$, with $n$ and $p$ positive integers, exploiting the notion of composite fermions (CF's). ${ }^{16}$ We discuss QH's and QE's at the highest ( $p$ th) level of the hierarchy if the $p /(2 n p+1)$ states are viewed as hierarchical states. In CF language this means that in addition to $p$ completely filled CF LL's there are CF's in the $(p+1)$ th CF LL (QE's) and empty states in the $p$ th CF LL (QH's).

Exact diagonalization data for $\mathrm{FQH}$ states for a few electrons on a sphere ${ }^{18,19}$ demonstrate a band structure of the energy levels. ${ }^{13,20,21}$ We use the observation-made for $n=p=1$ (near the $\frac{1}{3}$ state) - that the lowest band of levels is recovered correctly by the ("unprojected") CF picture ${ }^{20}$ to derive the diagonal ES parameters. We then verify these parameters numerically for larger values of $n$ and $p$. We also propose off-diagonal statistics parameters for which the ES counting (1) recovers correctly the number of states in first excited bands corresponding to the above configurations of QH's and QE's, for all the numerical data available.

Consider CF's, each carrying $2 n$ flux quanta, on a sphere. The total magnetic flux through the sphere affecting a $\mathrm{CF}$ is an integer number $2 S$ of quanta from the monopole charge minus $2 n\left(N_{e}-1\right)$ flux quanta bound to other CF's,

$$
2 S^{*}=2 S-2 n\left(N_{e}-1\right) .
$$

"Unprojected" CF's occupy the energy levels in the effective magnetic field determined by $2 S^{*}$. The relevant eigenstates are given by the monopole spherical harmonics $Y_{l M}^{\left(S^{*}\right)}$, where $l=S^{*}, S^{*}+1, \ldots$ numbers the "Landau levels" on the sphere, and $M$ taking values $-l, \ldots, l$ counts the degeneracies of these levels. ${ }^{22}$ When $p$ CF LL's are filled, $N_{e}=2 p S^{*}+p^{2}$, which results in the filling factor $\nu=N_{e} / 2 S \rightarrow p /(2 n p+1)$ as $S \rightarrow \infty$. 
If there are $N_{-}$QH's and $N_{+}$QE's,

$$
N_{e}=2 p S^{*}+p^{2}-N_{-}+N_{+} .
$$

Here a quasihole is an empty state in the $p$ th CF LL (with angular momentum $\left.l_{-}=S^{*}+p-1\right)$, a quasielectron is a $\mathrm{CF}$ in the $(p+1)$ th $\mathrm{CF}$ LL (with angular momentum $\left.l_{+}=S^{*}+p\right){ }^{23}$ The dimension of the Hilbert space for $N_{-}$ QH's and $N_{+}$QE's thus factors as

$$
W^{\prime}=\left(\begin{array}{c}
2 l_{-}+1 \\
N_{-}
\end{array}\right)\left(\begin{array}{c}
2 l_{+}+1 \\
N_{+}
\end{array}\right) \text {. }
$$

Comparing this [with $S^{*}$ determined by Eqs. (2) and (3)] with the ES counting of states (1) yields the dimensions of one-QH and one-QE states

$$
d_{-}=\frac{2 S+p+4 n}{2 n p+1}+p-1, \quad d_{+}=\frac{2 S+p}{2 n p+1}+p+1
$$

as well as the statistics matrix

$$
\begin{gathered}
g_{++}^{\prime}=2-g_{--}^{\prime}=1+2 n /(2 n p+1), \\
g_{-+}^{\prime}=-g_{+-}^{\prime}=2 n /(2 n p+1) .
\end{gathered}
$$

The lowest band of levels corresponds to the case where $\left|N_{+}-N_{-}\right|$in Eq. (3) takes its minimal possible value (so that at least one of the numbers $N_{-}$and $N_{+}$vanishes). Then Eq. (1) implies that the number of states in the band is completely determined by the diagonal ES parameters.

It has been demonstrated numerically near $\nu=\frac{1}{3}$ $(n=p=1)$ that the unprojected CF counting (4) [and correspondingly, the ES counting with parameters (6)] correctly recovers the number of states in the lowest band of levels. ${ }^{20}$ We have also verified parameters (6) for larger values of $p$. Examples are the spectra in Fig. 1. In Fig. 1(a), the lowest band of 21 states corresponds to 2 QH's near $\nu=\frac{1}{3}$. Figure 1(b) represents the spectrum which gives support to the diagonal statistics parameters (6) for larger values of $p$. The lowest band consists of a single multiplet of angular momentum $\frac{5}{2}$. The six states are recovered by the ES counting if they are viewed either as corresponding to one QE near $\nu=\frac{2}{5}$ $(n=1, p=2)$, or as corresponding to five QH's with respect to the state $\nu=\frac{3}{7}(n=1, p=3)$. For numerical support of the parameters (6) for larger values of $n$, see the discussion of Fig. 2 below for $\nu=\frac{1}{5}$.

Based on the above, we expect that the diagonal ES parameters derived from the unprojected CF picture (6) are generally valid; that is, $g_{++}=g_{++}^{\prime}$ and $g_{--}=g_{--}^{\prime}$. For Laughlin states $(p=1, m=2 n+1)$, parameters (6) reduce to those obtained in Refs. 12, 14, and 15.

Let us now turn to excited bands that are obtained from the lowest band by adding a pair $(1 \mathrm{QH}+1 \mathrm{QE})$. The full $\mathrm{CF}$ picture is obtained from the unprojected $\mathrm{CF}$ picture by the projection onto the lowest LL of the real magnetic field; the latter procedure eliminates some of the states counted in Eq. (4), as was shown numerically in various cases. ${ }^{20,21}$ To account for this, we modify the state counting (4), introducing additional "statistical interactions" between QH's and QE's, supposing that excitations of one kind affect the number of states available for excitations of the other kind as follows:
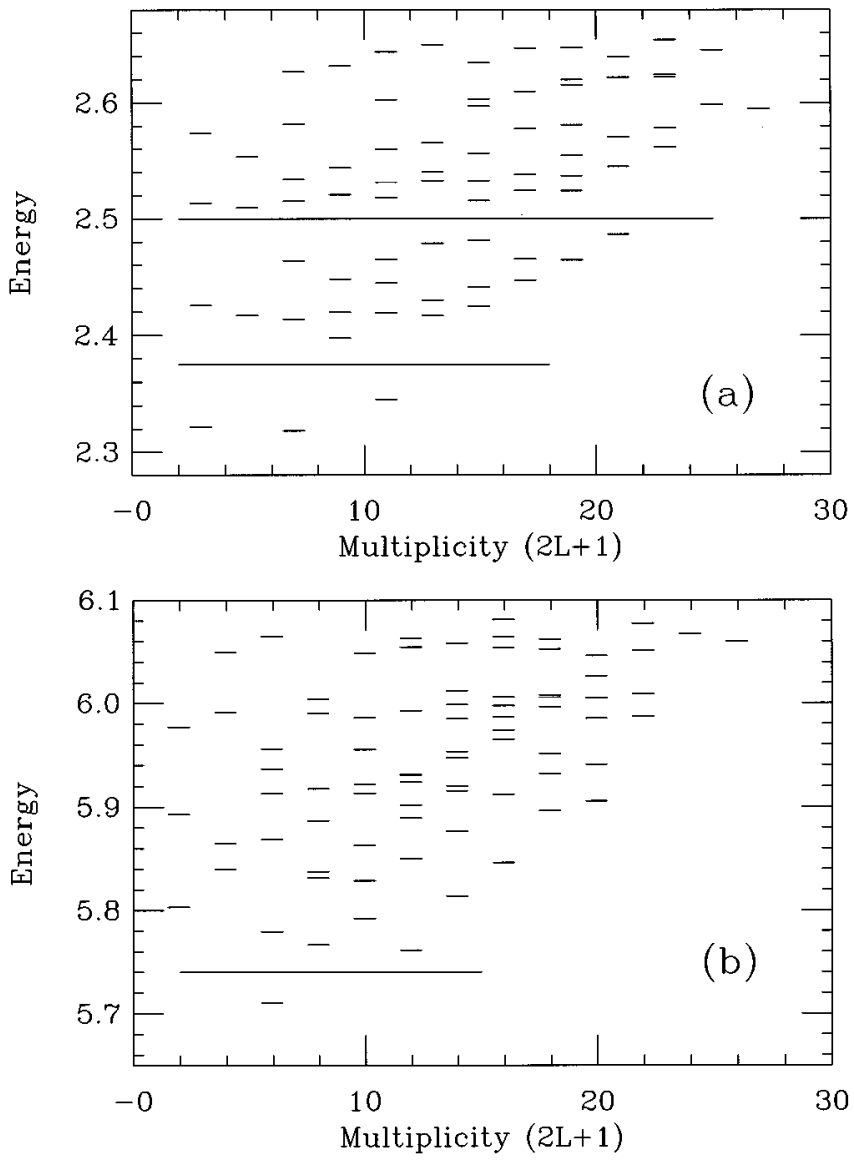

FIG. 1. Low-lying energy levels for electrons on a sphere: (a) $N_{e}=5$ and $2 S=14$. The solid lines separate the lowest band corresponding to two QH's (near $\nu=\frac{1}{3}$ ) from the first excited band (3 QH's +1 QE) and the latter band from higher states. (b) $N_{e}=7$ and $2 S=13$. The lowest "band" is a single 6-plet, whose dimension is obtained using diagonal ES parameters (6), with $p>1$.

$$
W=\left(\begin{array}{c}
2 l_{-}+1-\tilde{g}_{-+} N_{+} \\
N_{-}
\end{array}\right)\left(\begin{array}{c}
2 l_{+}+1-\tilde{g}_{+-} N_{-} \\
N_{+}
\end{array}\right) .
$$

This modifies the off-diagonal elements of the statistics matrix to

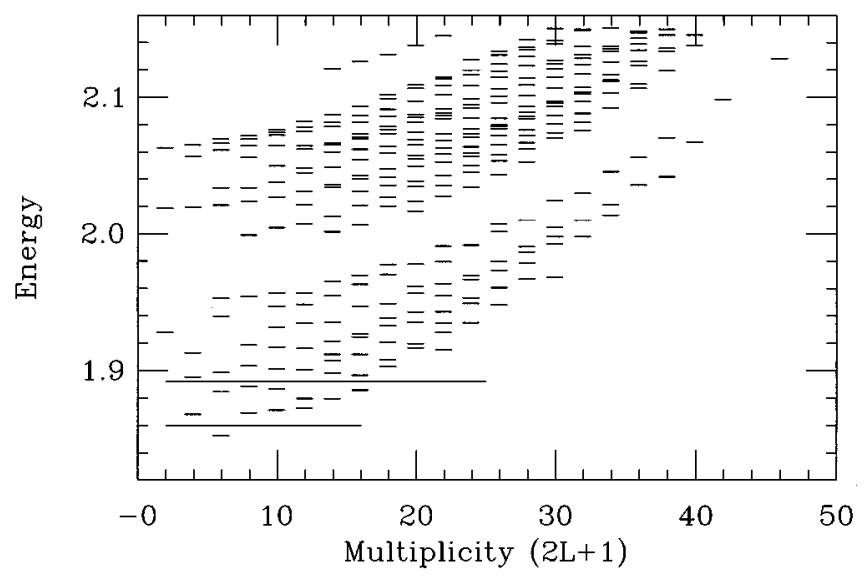

FIG. 2. Low-lying states for $N_{e}=5$ and $2 S=21$ (one QH near $\nu=\frac{1}{5}$ in the lowest band). The two lowest bands are counted correctly by the ES counting, with $n=2, p=1$. 
TABLE I. Neutral (1QE+1QH) bands above states $\nu=p /(2 n p+1)$, for various $N_{e}$ and $\nu$.

\begin{tabular}{lcccc}
\hline \hline$N_{e}$ & $2 S$ & $\nu$ & $L_{\max }$ & Ref. \\
\hline 6 & 15 & $\frac{1}{3}$ & 6 & 24 \\
7 & 18 & $\frac{1}{3}$ & 7 & 24 \\
8 & 21 & $\frac{1}{3}$ & 8 & 13 \\
9 & 24 & $\frac{1}{3}$ & 9 & 25 \\
5 & 20 & $\frac{1}{5}$ & 5 & 19 \\
5 & 28 & $\frac{1}{7}$ & 5 & 19 \\
8 & 16 & $\frac{2}{5}$ & 5 & 24 \\
10 & 21 & $\frac{2}{5}$ & 6 & 26 \\
9 & 16 & $\frac{3}{7}$ & 5 & 24 \\
12 & 23 & $\frac{3}{7}$ & 6 & 27 \\
\hline \hline
\end{tabular}

$$
g_{-+}=g_{-+}^{\prime}+\widetilde{g}_{-+}, \quad g_{+-}=g_{-+}^{\prime}+\widetilde{g}_{+-} .
$$

Consider first the case with $N_{-}=N_{+}=1$ in the first excited band [so that the lowest band is precisely the $p /(2 n p+1)$ state with zero angular momentum]. According to the unprojected $\mathrm{CF}$ picture, angular momenta of a $\mathrm{QE}$ and a $\mathrm{QH}\left(l_{+}\right.$and $\left.l_{-}\right)$differ by unity. Hence the angular momentum of the pair (coinciding with the total angular momentum of the state) may take values $L=1,2, \ldots, L_{\max }$, where $L_{\max }=l_{+}+l_{-}=N_{e} / p+p-1$.

Relevant numerical data are collected in Table I. The first neutral excited band always contains a single multiplet for each angular momentum $L=2,3, \ldots, L_{\max }$. As seen from Table I, the maximal angular momentum in the band is recovered correctly in the unprojected CF picture. The difference from the latter picture is in the minimal value of the total angular momentum, which is two (rather than one) for the system of interacting electrons. One can provide general arguments in support of the latter observation, ${ }^{27}$ note also that the projection onto the lowest LL in the CF picture eliminates the state with $L=1$, as was shown numerically for $\nu=\frac{1}{3} .{ }^{20}$

One can recover the correct number of states in the first neutral excited band, including the decomposition of states in angular momentum, within the ES counting. To show this, we formally introduce off-diagonal ES parameters depending on an integer number $\ell$, keeping their antisymmetry,

$$
g_{-+}(\ell)=g_{-+}^{\prime}+\ell, \quad g_{+-}(\ell)=g_{+-}^{\prime}-\ell,
$$

and consider the associated "partial" statistical weights [cf. Eq. (1)] corresponding to the statistics parameters

$$
W(\ell)=\left[d_{-}-g_{-+}(\ell)\right]\left[d_{+}-g_{+-}(\ell)\right] .
$$

It then follows from Eqs. (5)-(7) that $W(\ell)-W(\ell+1)$ $=2(\ell+1)+1$, and one can write

$$
W(\ell)=\sum_{L=\ell+1}^{L_{\max }}(2 L+1) .
$$

If one identifies $L$ with the angular momentum of the pair (equal to the total angular momentum), then formula (12) manifests the angular momentum decomposition in the ES counting: the difference $W(\ell)-W(\ell+1)$ counts states with angular momentum $L=\ell+1{ }^{28}$

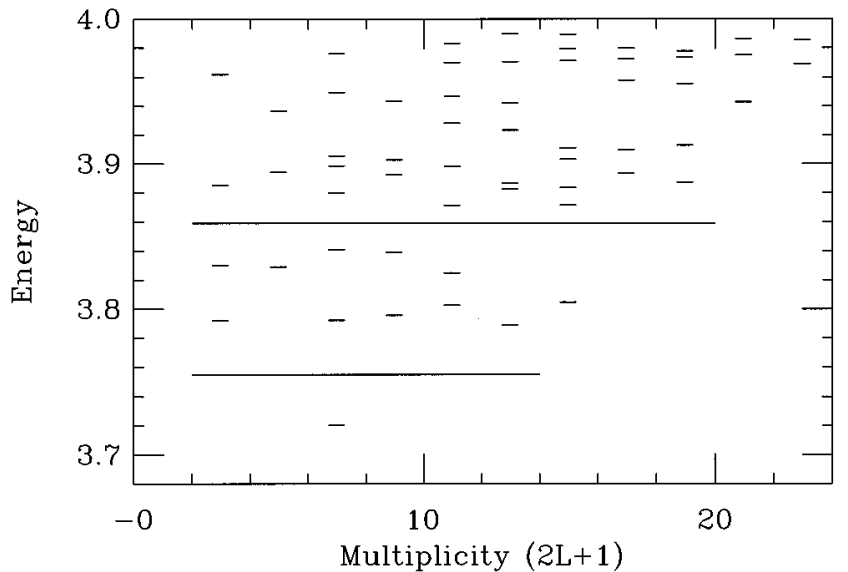

FIG. 3. Low-lying states for $N_{e}=6$ and $2 S=14$ : the first excited band corresponds to two different configurations of quasiparticles (see text).

We observe that $W(1)$ recovers the correct number of states in the lowest excited band. The corresponding offdiagonal statistics parameters are hence given by Eq. (10) with $\ell=1$. Comparing this with Eq. (9) then yields $\widetilde{g}_{-+}=-\widetilde{g}_{+-}=1$, thus finally resulting in the ES matrix

$$
g_{++}=g_{-+}=-g_{+-}=2-g_{--}=1+\frac{2 n}{2 n p+1} .
$$

For Laughlin states, the off-diagonal statistics parameters in Eq. (13) reduce to those proposed in Ref. 17.

The above logic entirely determines the set of statistics parameters $g_{\alpha \beta}$, using as input only the bands with a single species (thus determining the "diagonal" elements), and the first neutral excited bands with $N_{+}=N_{-}=1$. It is then of course of interest to try to apply these parameters to the other excited bands. Here we have to demand that the band should correspond to a unique configuration [the numbers $\left.\left(N_{-}, N_{+}\right)\right]$of quasiparticles (for the case involving several configurations, see discussion of Fig. 3 below). The above first neutral excited band, which we refer to as case (i), satisfies this requirement. Another case, which is referred to as case (ii), is the first excited band containing one QE and any number of QH's around the $1 / m$ state.

We have examined a large number of spectra for case (ii) around the $\frac{1}{3}$ state (some of which may be found in previously published spectra). ${ }^{13,21,29,17}$ Since case (ii) fixes $N_{+}=1$, we will simply list the cases examined as $\left(N_{e}, N_{-}\right)$(with $N_{-}$the number of QH in the first excited band): $\left(N_{e}=5, N_{-}=1,2,3,4\right) ; \quad\left(N_{e}=6, N_{-}=1,2,3,4\right)$; $\left(N_{e}=7, N_{-}=1,2,3\right)$. One of these bands $\left(N_{e}=5, N_{-}=3\right)$ is clearly seen in Fig. 1(a). In this, and in all other of these cases, the ES counting (1), as specified by Eqs. (5) and (13), correctly counts the states in the band, which fall below a well-defined (though small in a few cases) gap in the spectrum.

For FQH states with $n>1$, a band structure is expressed in the numerical data on a smaller energy scale. Thus, viewing the spectrum in Fig. 2 in terms of excitations around the $\frac{1}{5}$ state yields $N_{-}=1$ in the lowest band, and $N_{-}=2, N_{+}=1$ in the first excited band. The ES counting, 
using Eq. (13), then yields six states in the lowest band and 100 states in the first excited band. These two sets of states may be identified in Fig. $2 .^{30}$

As for case (i), the ES counting for case (ii) is in agreement with the full CF picture: it reproduces 165 states in the first excited band, obtained numerically in Ref. 21 (after the projection onto the lowest LL) for $N_{e}=6$ and $2 S=16$ $\left(N_{-}=2, N_{+}=1\right.$ around $\nu=\frac{1}{3}$ in the first excited band). The simple general formulas for the number of states in bands (ii) [e.g., in form (8)] thus call for an analytical understanding of the $\mathrm{CF}$ projection procedure, including underlying symmetry properties of the $\mathrm{CF}$ wave functions.

For cases other than (i) and (ii), the counting of states in the low-lying excited bands is more complicated. Consider, e.g., the case presented in Fig. 3. In the CF picture, the (degenerate) ground state has then the lowest LL filled, and one $\mathrm{CF}$ in the second LL. Therefore there are two distinct ways to form states in the first excited band (and consequently, two distinct configurations of quasiparticles relevant to this band), by exciting a CF (a) from the first to the second LL, or (b) from the second to the third LL. The total number of states in the band is expected to be the sum of two terms.

The ES counting, with Eqs. (13) and (5), applies to (a), yielding 84 states $\left(N_{+}=2, N_{-}=1\right)$, whereas the total number of states in the first excited band in Fig. 3 is 93. The difference (nine states) then represents a term of type (b), and this is precisely the Hilbert space dimension for a single CF in the third LL. ${ }^{31}$ The latter remark also shows the way to study other excited bands and, correspondingly, evaluate the density of excited states in energy for $\mathrm{FQH}$ states, using the ES counting. Work along these lines is in progress.

In conclusion, we have found the exclusion statistics parameters, both diagonal and off-diagonal, for QH's and QE's at the highest level of the hierarchy, for $\mathrm{FQH}$ states near $\nu=p /(2 n p+1)$. With these parameters, the ES counting of states applies to the first neutral excited band for the above FQH states on a sphere, as well as to the first excited bands containing one QE and any number of QH's around the $1 / m$ state. This provides general formulas for the number of states in the above bands, which are in agreement with numerical results given by the full ("projected") CF picture.

We thank Daniel Arovas, Heidi Kjønsberg, Jon Magne Leinaas, Stefan Mashkevich, Jan Myrheim, John Quinn, and Yong-Shi Wu for helpful discussions, and Piotr Sitko for sharing some unpublished results. S.B.I. and G.S.C. are grateful to the Center for Høyere Studier in Oslo, where this work was completed, for warm hospitality and support. G.S.C. was also supported in part by the NSF under Grant No. DMR-9413057, and M.D.J. by the NSF under Grant No. DMR-9301433. S.B.I. also acknowledges support from the Norwegian Research Council.
${ }^{1}$ The Quantum Hall Effect, 2nd ed., edited by R. E. Prange and S. M. Girvin (Springer, New York, 1990).

${ }^{2}$ Fractional Statistics and Anyon Superconductivity, edited by F. Wilczek (World Scientific, Singapore, 1990).

${ }^{3}$ J. M. Leinaas and J. Myrheim, Phys. Rev. B 37, 9286 (1988); Int. J. Mod. Phys. B 5, 2573 (1991).

${ }^{4}$ T. H. Hansson, J. M. Leinaas, and J. Myrheim, Nucl. Phys. B 384, 559 (1992).

${ }^{5}$ F. D. M. Haldane, Phys. Rev. Lett. 67, 937 (1991).

${ }^{6}$ S. B. Isakov, Int. J. Mod. Phys. A 9, 2563 (1994).

${ }^{7}$ S. B. Isakov, Mod. Phys. Lett. B 8, 319 (1994).

${ }^{8}$ Y. S. Wu, Phys. Rev. Lett. 73, 922 (1994).

${ }^{9}$ G. S. Canright and M. D. Johnson, J. Phys. A 27, 3579 (1994).

${ }^{10}$ A. Dasnières de Veigy and S. Ouvry, Phys. Rev. Lett. 72, 600 (1994).

${ }^{11}$ S. B. Isakov, S. Mashkevich, and S. Ouvry, Nucl. Phys. B 448, 457 (1995).

${ }^{12}$ M. D. Johnson and G. S. Canright, Phys. Rev. B 49, 2947 (1994).

${ }^{13}$ S. He, X. C. Xie, and F. C. Zhang, Phys. Rev. Lett. 68, 3460 (1992).

${ }^{14}$ D. Li and S. Ouvry, Nucl. Phys. B 430, 563 (1994).

${ }^{15}$ J. Yang, Phys. Rev. B 50, 11196 (1994).

${ }^{16}$ W. P. Su, Y. S. Wu, and J. Yang, Phys. Rev. Lett. 77, 3423 (1996).

${ }^{17}$ J. K. Jain, Phys. Rev. Lett. 63, 199 (1989); Phys. Rev. B 41, 7653 (1990); Science 266, 1199 (1994).

${ }^{18}$ F. D. M. Haldane, Phys. Rev. Lett. 51, 605 (1983).
${ }^{19}$ F. D. M. Haldane, in The Quantum Hall Effect (Ref. 1), Chap. 8.

${ }^{20}$ G. Dev and J. K. Jain, Phys. Rev. Lett. 69, 2843 (1992).

${ }^{21}$ X. G. Wu and J. K. Jain, Phys. Rev. B 51, 1752 (1995).

${ }^{22}$ T. T. Wu and C. N. Yang, Nucl. Phys. B 107, 365 (1976); Phys. Rev. D 16, 1078 (1977).

${ }^{23}$ If Jain's states with $\nu=p /(2 n p+1)$ are viewed as hierarchical states, this definition of QP's is apparently in agreement with the definition of QP's at the $p$ th level of the hierarchy.

${ }^{24}$ R. K. Kamilla, X. G. Wu, and J. K. Jain, Phys. Rev. Lett. 76, 1332 (1996); Phys. Rev. B 54, 4873 (1996).

${ }^{25}$ G. Fano, F. Ortolani, and E. Colombo, Phys. Rev. B 34, 2670 (1986).

${ }^{26}$ N. d'Ambrumenil and R. Morf, Phys. Rev. B 40, 6108 (1989).

${ }^{27}$ S. He, S. H. Simon, and B. I. Halperin, Phys. Rev. B 50, 1823 (1994).

${ }^{28}$ For $N_{+}>1$ and/or $N_{-}>1$, one can show the more general "nesting" property that $W\left(\ell^{\prime}>\ell\right)<W(\ell)$.

${ }^{29}$ P. Sitko, S. N. Yi, K. S. Yi, and J. J. Quinn, Phys. Rev. Lett. 76, 3396 (1996).

${ }^{30}$ Figure 2 may also be interpreted as excitations around $\nu=\frac{1}{3}$, for which one expects nine QH's and so 2002 states [using Eq. (1)]. These states are the entire lowest parabolic band in Fig. 2. Thus we see, in this figure, both "coarse" and "fine", structure in the energy bands, with the corresponding energy scales determined by the Coulomb pseudopotentials (Ref. 19) $V_{1}$ and $V_{3}$, respectively.

${ }^{31}$ We thank Y. S. Wu, who pointed out the latter observation to us. 